\title{
Onset dynamics of type A botulinum neurotoxin-induced paralysis
}

\author{
Frank J. Lebeda - Michael Adler • \\ Keith Erickson - Yaroslav Chushak
}

Received: 19 December 2007 / Accepted: 20 March 2008 / Published online: 13 June 2008

(C) to the authors 2008

\begin{abstract}
Experimental studies have demonstrated that botulinum neurotoxin serotype A (BoNT/A) causes flaccid paralysis by a multi-step mechanism. Following its binding to specific receptors at peripheral cholinergic nerve endings, BoNT/A is internalized by receptor-mediated endocytosis. Subsequently its zinc-dependent catalytic domain translocates into the neuroplasm where it cleaves a vesicle-docking protein, SNAP-25, to block neurally evoked cholinergic neurotransmission. We tested the hypothesis that mathematical models having a minimal number of reactions and reactants can simulate published data concerning the onset of paralysis of skeletal muscles induced by BoNT/A at the isolated rat neuromuscular junction (NMJ) and in other systems. Experimental data from several laboratories were simulated with two different models that were represented by sets of coupled, first-order differential equations.
\end{abstract}

Electronic supplementary material The online version of this article (doi:10.1007/ s10928-008-9087-3 ) contains supplementary material, which is available to authorized users.

F. J. Lebeda $(\bowtie)$

Integrated Toxicology Division, US Army Medical Research Institute of Infectious Diseases, 1425 Porter Street, Fort Detrick, MD 21702-5011, USA

e-mail: Frank.Lebeda@ amedd.army.mil

M. Adler

US Army Medical Research Institute of Chemical Defense, Aberdeen Proving Ground, MD 21010-5400, USA

\section{K. Erickson}

Department of Mathematical Sciences, US Military Academy, West Point, NY 10996, USA

Y. Chushak

Biotechnology High Performance Computing Software Applications Institute, US Army Medical Research and Materiel Command, Fort Detrick, MD 21702-5011, USA

Y. Chushak

Wright-Patterson AFB, Dayton, OH 45433, USA 
In this study, the 3-step sequential model developed by Simpson (J Pharmacol Exp Ther 212:16-21,1980) was used to estimate upper limits of the times during which anti-toxins and other impermeable inhibitors of BoNT/A can exert an effect. The experimentally determined binding reaction rate was verified to be consistent with published estimates for the rate constants for BoNT/A binding to and dissociating from its receptors. Because this 3-step model was not designed to reproduce temporal changes in paralysis with different toxin concentrations, a new BoNT/A species and rate $\left(k_{S}\right)$ were added at the beginning of the reaction sequence to create a 4 -step scheme. This unbound initial species is transformed at a rate determined by $\mathrm{k}_{S}$ to a free species that is capable of binding. By systematically adjusting the values of $\mathrm{k}_{\mathrm{S}}$, the 4-step model simulated the rapid decline in NMJ function $\left(k_{S} \geq 0.01\right)$, the less rapid onset of paralysis in mice following i.m. injections $\left(k_{S}=0.001\right)$, and the slow onset of the therapeutic effects of BoNT/A $\left(k_{S}<0.001\right)$ in man. This minimal modeling approach was not only verified by simulating experimental results, it helped to quantitatively define the time available for an inhibitor to have some effect $\left(t_{\text {inhib }}\right)$ and the relation between this time and the rate of paralysis onset. The 4-step model predicted that as the rate of paralysis becomes slower, the estimated upper limits of $\left(\mathrm{t}_{\text {inhib }}\right)$ for impermeable inhibitors become longer. More generally, this modeling approach may be useful in studying the kinetics of other toxins or viruses that invade host cells by similar mechanisms, e.g., receptor-mediated endocytosis.

Keywords In vitro data $\cdot$ In vivo data $\cdot$ Clinical data $\cdot$ Toxicodynamics . Stimulation-frequency dependence

Manuscript Classification Animal pharmacokinetics/pharmacodynamics . Human pharmacodynamics · Simulation

\section{Abbreviations}

$\begin{array}{ll}\text { BoNT/A } & \text { Botulinum neurotoxin type A } \\ \text { NMJ } & \text { Neuromuscular junction } \\ \mathrm{t}_{10} & \text { Time-to- } 10 \% \text { peak tension } \\ \mathrm{t}_{\text {inhib }} & \text { Time at which } \geq 10 \% \text { of unbound BoNT/A is present and available } \\ & \text { for an inhibitor to exert some effect }\end{array}$

\section{Introduction}

The neurotoxins from Clostridium botulinum and several related species represent some of the most lethal substances known [1-3]. The signs and symptoms include flaccid paralysis of the voluntary muscles, respiratory distress and death. The onset times and durations of paralysis depend on the serotype involved, the exposure route and the intoxicating dose. As summarized in [4], the public is becoming increasingly aware of the roles of botulinum neurotoxins as food poisoning agents, as potential bioweapons $[1,2,5,6]$, and as approved treatments for various neurologic indications and other clinical uses [7]. Significant resources [8,9] have been devoted to the largescale production of heptavalent botulism antitoxin [10]. Complementary research to engineer and develop high-affinity, monoclonal neutralizing antibodies is also being conducted [11]. 
The bacteria express these toxins as single chain polypeptides (MW $\sim 150 \mathrm{kDa}$ ) which are later post-translationally modified to form two chains (heavy, $100 \mathrm{kDa}$ and light, $50 \mathrm{kDa}$ ) that are covalently linked by a disulfide bridge. The C-terminal half of the heavy chain specifically binds to extracellular acceptors at peripheral cholinergic nerve terminals [12] that innervate striated and smooth muscles. A process resembling receptor-mediated endocytosis internalizes the toxin-bound receptor. As the intravesicular environment becomes acidic ( $\mathrm{pH} 5$ ), the N-terminal half of the heavy chain helps form cation-selective channels that may be involved in allowing the escape of the toxic moiety (presumably the catalytic light chain or its derivatives) into the neuroplasm (reviewed in [13]). The toxic fragment is a zinc-dependent protease that cleaves at distinct sites and in a serotype-specific manner one or more of the SNARE proteins (SNAP-25, syntaxin and VAMP) involved in the synaptic vesicle-mediated release of acetylcholine. Once internalized, BoNT is no longer susceptible to circulating neutralizing antibodies or other impermeable inhibitors of its toxicity. This homologous family of proteins are grouped into seven immunologically distinct serotypes (BoNT/A-G) [3,14]. SNAP-25 is cleaved by BoNT serotypes A, E and C1, syntaxin is cleaved by BoNT/C1, and VAMP is cleaved by the remaining BoNT serotypes [14].

The present study was designed to extend a data-driven minimal model developed by Simpson [15] that described the kinetics of botulinum neurotoxin serotype A (BoNT/A) at the neuromuscular junction (NMJ) in producing paralysis in vitro. This original deterministic model consisted of a sequence of reactions based on the known mechanism of BoNT/A action, namely, binding to specific receptors located at cholinergic nerve terminals, translocating into the neuroplasm and, in turn, exerting a toxic effect. All three steps were separately examined experimentally and quantitatively characterized by apparent first-order reaction rates. Modifications were introduced in our study to allow for the changes in paralysis time course seen under different in vivo conditions [16-18]. We also developed a quantitative relationship between the onset rate of paralysis and the time that is available to neutralizing antitoxins or other non-permeable countermeasures to exert some inhibitory effect.

\section{Methods}

Reaction rates and other rate constants

The experimentally measured reaction rate of BoNT/A binding to the in vitro NMJ preparation model was compared to previously determined association and dissociation (on and off) rate constants reported in [19] for rat brain synaptosomes. These microscopic rate constants were adjusted for a $\sim 20^{\circ} \mathrm{C}$ difference in temperature because the NMJ experiments were conducted at $35^{\circ} \mathrm{C}$ and the synaptosomal studies were performed at $4^{\circ} \mathrm{C}$. The experimentally determined value for the temperature coefficient, $\mathrm{Q}_{10}$, for the binding step was used as the multiplicative factor by which a rate constant is increased when increasing the temperature by $10^{\circ} \mathrm{C}$ [20]. It was assumed that the measured $\mathrm{Q}_{10}$ value was a constant for this $20^{\circ} \mathrm{C}$ range of temperatures, that a steady state of equilibrium was achieved and that toxin was not internalized following binding. 
The present models for the receptor binding, the nerve-terminal entry and the subsequent toxic (lytic) activity of BoNT/A were developed using the same general reaction schemes and numerical procedures that were previously used for modeling the kinetics of neurotransmission at peripheral and central synapses [21,22]. Reactions describing the 3-step model were derived from [15] for BoNT/A intoxication at the in vitro rat $\mathrm{NMJ}$ :

$$
\text { free } \stackrel{k_{B}}{\rightarrow} \text { bound } \stackrel{k_{T}}{\rightarrow} \text { trans } \stackrel{k_{L}}{\rightarrow} \text { lytic }
$$

where the system of coupled first-order differential equations is:

$$
\begin{aligned}
& d[\text { free }] / d t=-k_{B}[\text { free }] \\
& d[\text { bound }] / d t=k_{B}[\text { free }]-k_{T}[\text { bound }] \\
& d[\text { trans }] / d t=k_{T}[\text { bound }]-k_{L}[\text { trans }] \\
& d[\text { lytic }] / d t=k_{L}[\text { trans }] .
\end{aligned}
$$

This 3-step model represents a minimal version of the original model for a free amount of neurotoxin to bind, translocate into the neuroplasm and generate lytic activity [15]. Each species of BoNT/A is associated with a different environment: extracellular in solution (free), on the surface, bound to a receptor (bound), intracellular endocytotic vesicle (trans), and intracellular neuroplasm (lytic). The three irreversible steps eventually lead to the blockade of vesicle-mediated neurotransmission and the development of complete muscle paralysis in the NMJ experiments [15]. The binding step implicitly contains diffusion of free BoNT/A $(0.1 \mathrm{nM})$ to its binding sites at synaptic termini. The bound toxin undergoes translocation into the neuroplasm. Intracellularly, the toxic moiety exerts it enzymatic proteolytic activity on SNAP-25 [14]. Embedded in this species is the paralysis that occurs postsynaptically. Rates of reaction were taken from [15] where $k_{B}=0.058 \mathrm{~min}^{-1}, k_{T}=0.141 \mathrm{~min}^{-1}$ and $k_{L}=0.013 \mathrm{~min}^{-1}$. This minimal 3 -step scheme is a simplification from [15] because it omits three unknown parameters representing binding sites for each species of toxin. An analytic expression derived for the lytic species,

$$
\begin{aligned}
& \operatorname{lytic}(t)=[T]_{\text {tot }} \\
& \left(1-\frac{\left(e^{-k_{T} t} k_{B} k_{L}\left(k_{B}-k_{L}\right)-e^{-k_{L} t} k_{B} k_{T}\left(k_{B}-k_{T}\right)+e^{-k_{B} t} k_{L} k_{T}\left(k_{L}-k_{T}\right)\right)}{\left(k_{B}-k_{L}\right)\left(k_{B}-k_{T}\right)\left(k_{L}-k_{T}\right)}\right),
\end{aligned}
$$

was used to verify the selection of the time steps for the numerical solution and to establish further confidence in our minimal approach. $[T]_{\text {tot }}$ represents the total normalized amount of toxin. Analytic expressions for the other species are available from the Electronic Supplementary Material. 
Reaction equations: 4-Step Model

The 4-step model is an extension of the 3-step model. BoNT/A is present at $\mathrm{t}=0$ in the bulk solution $(\mathrm{S})$ and the rate constant, $k_{S}$, simulates the rate of movement of the non-binding species of BoNT/A from the bulk medium to the extracellular solution that surrounds the nerve termini:

$$
\begin{gathered}
\text { bulk } \stackrel{k_{S}}{\rightarrow} \text { free } \stackrel{k_{B}}{\rightarrow} \text { bound } \stackrel{k_{T}}{\rightarrow} \text { trans } \stackrel{k_{L}}{\rightarrow} \text { lytic } \\
d[\text { bulk }] / d t=-k_{S}[\text { bulk }] \\
d[\text { free }] / d t=k_{S}[\text { bulk }]-k_{B}[\text { free }]
\end{gathered}
$$

In the 3-step model, initial conditions $(t=0)$ for the normalized molecular species of BoNT were $[$ free $]=1.0,[$ bound $]=0$ and $[$ lytic $]=0$, while in the 4-step model these species were initially set to zero and the $[$ bulk $]=1.0$. As in the 3-step model, an analytic expression was derived for the lytic species:

lytic $(t)=[T]_{\text {tot }}-\frac{[T]_{\text {tot }}\left(\begin{array}{l}e^{-k_{T} t} k_{B} k_{L} k_{S}\left(k_{B}-k_{L}\right)\left(k_{B}-k_{S}\right)\left(k_{L}-k_{S}\right)- \\ e^{-k_{S} t} k_{B} k_{L} k_{T}\left(k_{B}-k_{L}\right)\left(k_{B}-k_{T}\right)\left(k_{L}-k_{T}\right)+ \\ e^{-k_{L} t} k_{B} k_{S} k_{T}\left(k_{B}-k_{S}\right)\left(k_{B}-k_{T}\right)\left(k_{S}-k_{T}\right)- \\ e^{-k_{B} t} k_{L} k_{S} k_{T}\left(k_{L}-k_{S}\right)\left(k_{L}-k_{T}\right)\left(k_{S}-k_{T}\right)\end{array}\right)}{\left(k_{B}-k_{L}\right)\left(k_{B}-k_{S}\right)\left(k_{L}-k_{S}\right)\left(k_{B}-k_{T}\right)\left(k_{L}-k_{T}\right)\left(k_{S}-k_{T}\right)}$.

Analytic expressions for the other species in this model are available from the Electronic Supplementary Material. Values of $k_{S}$ were systematically changed from 0.0001 to $15 \mathrm{~min}^{-1}$. In these models of in vivo systems there is no explicit clearance (i.e., loss of toxin from those systems), only an implicit one that is embedded in the $\mathrm{k}_{S}$ rate value. It was assumed that the amount of BoNT that was injected intramuscularly became distributed in the extra-vascular space, and was partially taken up by the circulatory system or in various organs (e.g., liver, spleen, kidney) as has been demonstrated in [4] following i.v. injections of BoNT. The kinetic parameters used in these minimal models are tabulated at http://botdb.abcc.ncifcrf.gov.

Diaphragm muscle function (peak twitch amplitude induced by electrical stimulation of the phrenic nerve) was defined as the normalized amount of tension remaining after the formation of the lytic species:

$$
\text { tension }=1.0-[l y t i c]
$$

Values of $\mathrm{t}_{10}$ are the times at which the model predicts that the peak twitch tension is $10 \%$ of the maximum tension, i.e., a $90 \%$ reduction in tension. This level of residual tension was used in these in vitro experiments because complete paralysis is difficult to accurately measure [15]. The twitch responses were measured after exposure to $300 \mathrm{ng}$ $\left(10^{-10} \mathrm{M}\right)$ BoNT/A in $20 \mathrm{ml}$ bathing chambers as in previous in vitro NMJ studies [23]. Nerve-muscle preparations were exposed to toxin-containing media that were made 
with $0.5 \mathrm{mM} \mathrm{Ca}^{2+}$ and $5.0 \mathrm{mM} \mathrm{Mg}^{2+}$ to prevent toxin internalization. After a $60 \mathrm{~min}$ incubation, the preparations were washed with saline then soaked in control medium.

Other response measures were used in the mouse in vivo studies [7,17] and in clinical studies [18]. In [7], time spent by mice on an exercise wheel was monitored during the onset and recovery of the BoNT/A-induced paralysis $(0.2-1.5 \mathrm{ng})$. The study in [17] measured toe abduction reflexes as the functional parameter affected by the i.m. injection of $26 \mathrm{ng}$ of BoNT/A. In the clinical study [18], the patient reported the alleviation of subjective symptoms of cervical dystonia following i.m. injections of BoNT/A which are 1-4 ng according to [24] for this neurologic disorder. In all of the simulations using these in vivo data, the term "response" replaces "tension" in Eq. 3.

In this study, we estimated the upper limit of time that non-permeable inhibitors of BoNT/A (e.g., an antibody) have some effect. The value of this available time ( $\mathrm{t}_{\text {inhib }}$ ) was operationally defined as the time at which the amount of unbound BoNT/A is at least $10 \%$ of the initial unbound species. We are using this metric from the point of view that it is the toxin that needs to be inhibited by an antibody or other non-permeable inhibitor. Because $t_{\text {inhib }}$ is quantitative, it can be later refined when the time course of the unbound amount of BoNT/A is experimentally resolved. In the 3-step model, the relative value of the unbound BoNT/A is equal to the value of the free species, while in the 4-step model, the relative value of the unbound BoNT/A is equal to the sum of the values of the free and the bulk species.

\section{Computer programs and statistical methods}

The kinetic schemes were visually constructed and edited interactively using JDesigner which is part of the open source Systems Biology Workbench (SBW) [25]. This program saves models in SBML format so that they can be used as a front-end software component to any SBW-compatible equation solver. The ordinary first-order differential equations describing the reactions built with JDesigner were solved by Jarnac, a script language-based program for analyzing biochemical reaction networks

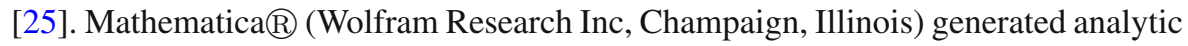
expressions that were used to verify the selection of the time steps for the numerical solutions by Jarnac and to establish further confidence in our minimal approach. A spreadsheet-compatible data-analysis utility was used to digitize and store twodimensional coordinates of data points from selected figures shown in [4,7,15-17] and [18] (Datatrend Software, Raleigh, North Carolina). Linear regression analysis of the form $y=y_{0}+a x$ was performed on the data in Fig. 2 to calculate coefficients of determination $\left(\mathrm{R}^{2}\right)$ and values of the slope (a) and intercept $\left(\mathrm{y}_{0}\right)$ along with standard errors of the mean (S.E.M.) using SigmaPlot (ver. 9.01, 2004, Systat Software, Inc., Chicago, Illinois). Area under the curve (AUC) calculations were performed on the numerical outputs (Fig. 1) by summing the amount of free toxin (ng units) present at each time increment $(60 \mathrm{~min})$ in the 3-step model. Because of the small amount of free toxin predicted for the simulation in Fig. 4, only the amount of bulk toxin was summed at each time increment ( $1 \mathrm{~min}$ ) in the 4-step model. Elimination rates of the unbound (i.e., free or bulk) toxin species were calculated by taking the amount (ng units) at $\mathrm{t}=0$ and dividing by the AUC value (min-ng units). 


\section{Results}

Relating the binding reaction rate to the receptor binding and dissociation rate constants for BoNT/A

Before examining the 3-step model, we tested the hypothesis that the receptors for BoNT/A are not saturated at $0.1 \mathrm{nM}$, the concentration used in [15]. In addition, we evaluated the relation between the measured apparent first-order binding reaction at the rat NMJ $\left(k_{B}\right)$ [15] and the association $\left(k_{o n}\right)$ and dissociation $\left(k_{o f f}\right)$ microscopic rate constants that have been measured using rat cerebrocortical-derived, synaptosome preparations [19]. The values for $k_{o n}=0.0138 \mathrm{nM}^{-1} \mathrm{~min}^{-1}$ and $k_{\text {off }}=0.0072 \mathrm{~min}^{-1}$ at $4^{\circ} \mathrm{C}$ [19] correspond to a $K_{d}=0.52 \mathrm{nM}$. [15] the rate of paralysis in the rat NMJ preparation $\left(k_{B}=0.058 \mathrm{~min}^{-1}\right.$ was measured under conditions of low $\left[\mathrm{Ca}^{2+}\right]$, high $\left[\mathrm{Mg}^{2+}\right]$ containing solutions at $35-36^{\circ} \mathrm{C}$ to block the internalization step (Fig. 2 of [15])). In keeping with experimental observations [15], it was assumed that low $\left[\mathrm{Ca}^{2+}\right]$, high $\left[\mathrm{Mg}^{2+}\right]$-containing solutions did not affect these on and off rate constants in this reversible reaction:

$$
U+R \underset{k_{\text {off }}}{\stackrel{k_{\text {on }}}{\rightleftarrows}} B
$$

where $[U]=[$ BoNT/A $]$ (unbound), $[R]=$ binding site (productive ecto-acceptor) $[26]$, and $[B]=[$ BoNT/A $]$ (bound). Using the experimentally determined value for $\mathrm{Q}_{10}=1.6$ for the binding step [15], the temperature-adjusted values for $k_{\text {on }}$ and $k_{\text {off }}$ at $35^{\circ} \mathrm{C}$ were estimated to be $0.056 \mathrm{nM}^{-1} \mathrm{~min}^{-1}$ and $0.029 \mathrm{~min}^{-1}$, respectively, and are used in the equations below. The total toxin and binding site concentrations at $\mathrm{t}>0$ are

$$
[T]_{t o t}=[U]+[B] ;[R]_{t o t}=[R]+[B] .
$$

The binding-site occupancy was assumed to be described by the mass-law formulation to give an equilibrium dissociation constant

$$
K_{d}=\frac{k_{o f f}}{k_{o n}}=\frac{[U][R]}{[B]}
$$

in which the species concentrations are substituted for their activities. These terms are related to the experimentally measured value for $k_{B}$, an apparent first-order rate constant, that is the reciprocal of the relaxation time constant $(\tau)$ for a reversible, single-step bimolecular reaction [27]:

$$
k_{B}=1 / \tau=\left([T]_{t o t}+[R]_{t o t}\right) k_{o n}+k_{o f f}
$$

Combining and rearranging Eqs. 4-6 yields a quadratic expression

$$
[R]^{2}+\left\{2 K_{d}-k_{B} / k_{o n}\right\}[R]+\left(K_{d}[T]_{t o t}-K_{d} k_{B} / k_{o n}+K_{d}^{2}\right)=0
$$


in which the positive root of $[R]$ is:

$$
[R]=\frac{-\left\{2 K_{d}-k_{B} / k_{o n}\right\}+\sqrt{\left.\left\{2 K_{d}-k_{B} / k_{o n}\right)\right\}^{2}+4\left\{K_{d}[T]_{t o t}-K_{d} k_{B} / k_{\text {on }}+K_{d}^{2}\right\}}}{2}
$$

Solving for $[R]$ allows values for $[U]$ and $[B]$ to be calculated

$$
\begin{aligned}
& {[U]=\frac{\left([T]_{t o t} / K_{d}\right)}{[R]+\left(1 / K_{d}\right)}} \\
& {[B]=[T]_{t o t}-[U] .}
\end{aligned}
$$

Using this approach with a dimensionless value of $\mathrm{T}_{\text {tot }}=1.0$, the values for the reaction species at $35^{\circ} \mathrm{C}$ (assuming a steady state of equilibrium and no toxin internalization) were calculated to be $[R]=8.6,[U]=0.69$ and $[B]=0.31$. From these results, the values of $K_{d}$ and the corresponding on and off rate constants support the idea that the binding sites for BoNT/A are not saturated. These results also support the hypothesis that the binding reaction rate for BoNT/A measured in the isolated NMJ at $35-36^{\circ} \mathrm{C}$ can be feasibly compared with the microscopic on/off rate constants for BoNT/A measured in rat brain at $4^{\circ} \mathrm{C}$.

The 3-step model: estimates of $t_{\text {inhib }}$ and the frequency dependence of toxicity at the NMJ

As depicted in Fig. 1, the experimental data collected from the in vitro NMJ (adapted from Fig. 4 of [15]) was simulated with a minimal 3-step model. In those experiments, BoNT/A was incubated in low- $\mathrm{Ca}^{2+}$, high- $\mathrm{Mg}^{2+}$ containing-media for 40 min to allow near-maximal binding. A second incubation period with normal media allowed for near-maximal translocation ( $~ 90 \%$ in $16 \mathrm{~min}$ ) which was followed by the commencement of phrenic nerve stimulation to monitor the gradual decline in twitch tension. In contrast, BoNT/A was applied in this and the 4-step model at $\mathrm{t}=0$. The time courses of the four BoNT/A species can be visually compared during the development of BoNT/A-induced paralysis (time-to-10\% tension, $\mathrm{t}_{10}=204 \mathrm{~min}$ ). The numerical solution generated by Jarnac for the tension curve using a time step of 1 min (Fig. 1, black line) was verified with an analytic expression (white curve superimposed on black line, Eqs. 1e and 3). The time course of the free BoNT/A species provides a quantitative metric for estimating the time available for an inhibitor to exert some effect $\left(\mathrm{t}_{\mathrm{inhib}}\right)$, as defined in the Methods. In this 3-step model, $\mathrm{t}_{10}$ was predicted to be

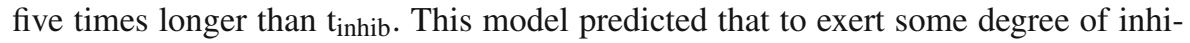
bition, impermeable neutralizing antibodies or BoNT/A inhibitors need to be applied within $40 \mathrm{~min}$ of the addition of $0.1 \mathrm{nM}$ BoNT/A to the bathing chamber, i.e., before a $\sim 20 \%$ reduction of nerve-evoked twitch tension occurs.

The use-dependence associated with BoNT/A at the NMJ (data adapted from Fig. 5 of [15]) is illustrated in Fig. 2A in which paralysis times decreased (smaller values of 


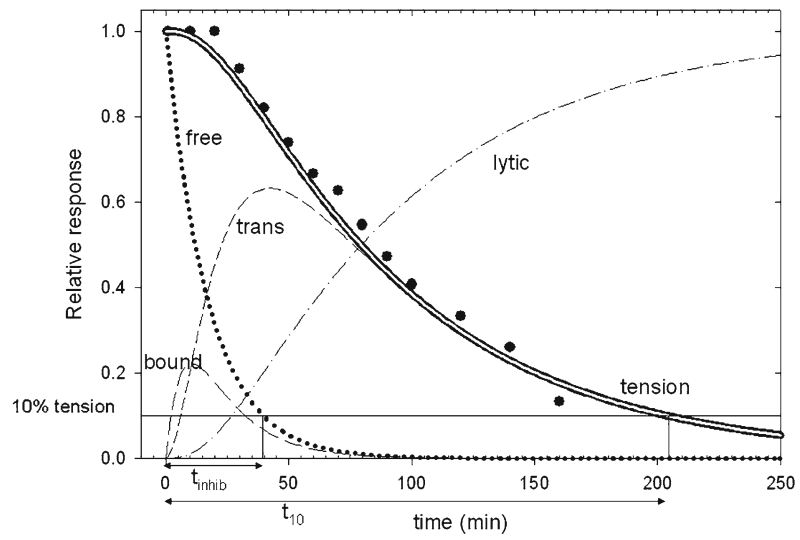

Fig. 1 Simulation of experimental NMJ data with the 3-step model. The time courses of the model species can be compared with the development of BoNT/A-induced paralysis (filled symbols, data from [15]). The time-to-10\% tension, $\mathrm{t}_{10}$, was $204 \mathrm{~min}$. The upper limit for the amount of time available for an inhibitor of BoNT/A to exert some effect, $t_{\text {inhib, }}$ was $\leq 40 \mathrm{~min}$ (see Methods). All BoNT/A species are represented as relative values. Dotted line: free BoNT/A; long dashes: bound toxin; short dashes: translocated species; black line: amplitudes of peak twitch tension numerically calculated from the 3-step model; white line (superimposed on black line): amplitudes of peak twitch tension calculated from Eq. 1e

$\mathrm{t}_{10}$ ) as the frequency of stimulating the phrenic nerve increased. Results of the linear regression analysis (see Methods) were:

$$
t_{10}=94.6(5.4 \text {, S.E.M })-85.4(35.9 \text {, S.E.M. }) \text { frequency }\left(R^{2}=0.73\right)
$$

Changing the values of either $k_{B}$ or $k_{T}$ could only partially simulate the experimental data. In contrast, altering the values for the third reaction rate $\left(k_{L}\right)$ could, for the first time to our knowledge, simulate the entire set of data associated with the frequencydependent enhancement of BoNT/A-induced paralysis. Values for $\mathrm{t}_{10}$ (Fig. 2B) overlap with the experimental data points that reside within a linear portion of the predicted non-linear relation between values of $k_{L}$ and normalized times-to-10\% tension. The values of $k_{L}\left(0.013-0.019 \mathrm{~min}^{-1}\right)$ correspond to values of stimulation frequencies that are within practical experimental limits (Fig. 2C):

$$
\text { frequency }=0.54(0.67 \text {, S.E.M. })+43.25(4.16 \text {, S.E.M. }) k_{L}\left(R^{2}=0.98\right) \text {. }
$$

The 4-step model: in vitro NMJ versus in vivo systems and estimates for $t_{\text {inhib }}$

Altering the three original reaction rates $\left(k_{B}, k_{T}\right.$ and $\left.k_{L}\right)$ failed to produce the slower onset rates of BoNT/A-induced paralysis observed in vivo. Thus, the 3-step model examined above was extended and used to simulate BoNT/A-induced responses of patients when administered as an approved therapeutic product [18] and of mice when injected as a local paralysant $[7,17]$. In this model, a new, initial reaction rate $\left(k_{S}\right)$ was created that represents the movement of BoNT/A (bulk species) from distal locations 
Fig. 2 Different values of $k_{L}$ in the 3-step model simulated the frequency-dependence of the rate of onset of BoNT/A-induced paralytic activity. Panel A: plot of data points where values for $\mathrm{t}_{10}$ are expressed as normalized values. Panel B: The 3-step model (dashed line) produced a non-linear relation between $k_{L}\left(0.01-0.3 \mathrm{~min}^{-1}\right)$ and the time-to- $10 \%$ tension, $\mathrm{t}_{10}$. Within the range of experimental data, the plot was linear and at higher values of $k_{L}$ approached an asymptotic value for the normalized values of $\mathrm{t}_{10}$. Panel C: Values of $k_{L}$ were adjusted to simulate normalized $t_{10}$ values at each of the four experimentally used nerve stimulation frequencies. Circles: experimental data from Fig. 4 in [15]; lines in Panels A and C: least-squares fits drawn through the data points
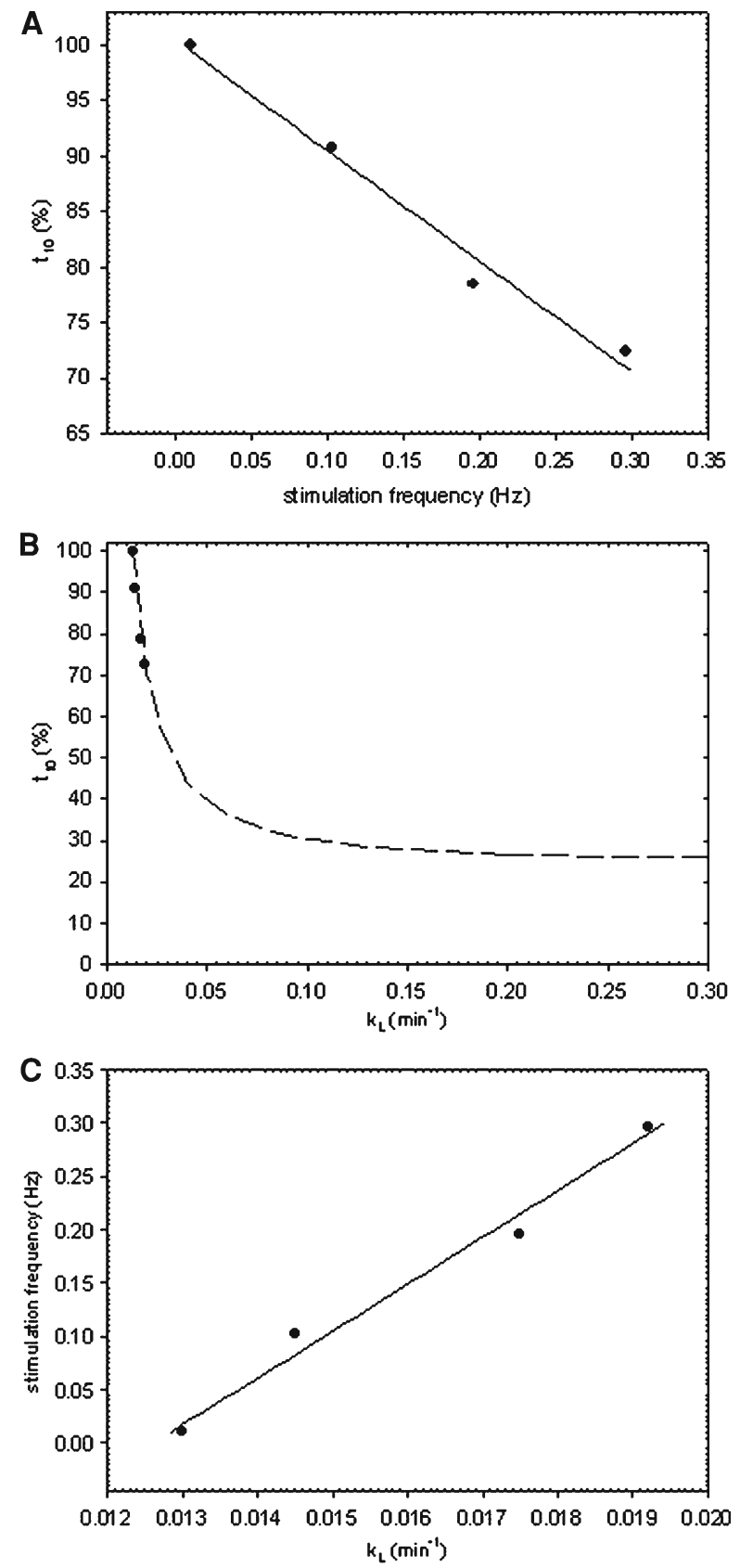

from its receptors to the proximal volume where the BoNT/A (free species) is available for binding.

Slow onset kinetics for the beneficial effects of treatments using BoNT/A have been published in the clinical literature. Normalized clinical data represented in 


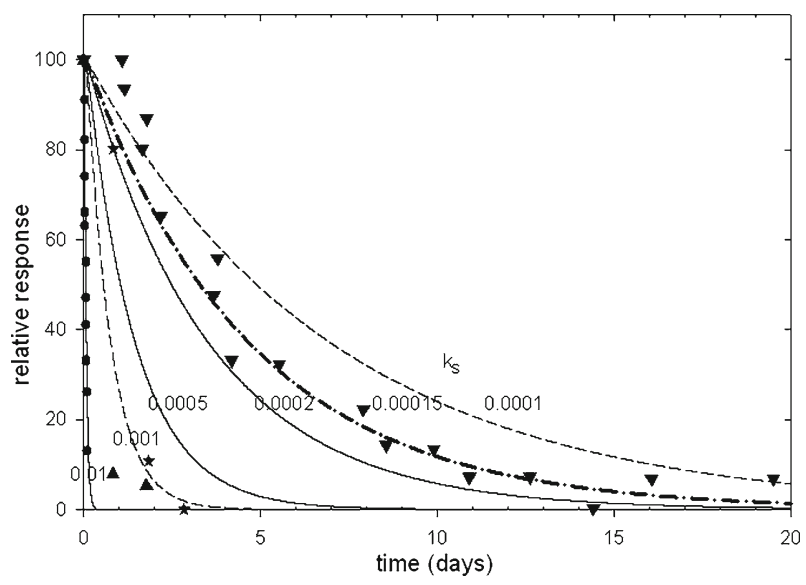

Fig. 3 Different values of $k_{S}$ in the 4-step model simulated the onset of BoNT/A-induced effects in a variety of in vitro and in vivo data sets. The onset of paralysis with bath-applied BoNT/A at the rat NMJ (circles, from Fig. 1) is rapid and is associated with $k_{S}$ values $\geq 0.01 \mathrm{~min}^{-1}$. In vivo data (time using an exercise wheel, see Methods) from locally injected mice (stars, data from [17]; upward triangles, data from [7]) were simulated using $k_{S}=0.001 \mathrm{~min}^{-1}$. Clinical data (subjective measure of relief of neurologic symptoms, see Methods; downward triangles) are from a patient whose first session of BoNT/A treatment was on day 0 (data from [18]). The maximum BoNT/A-induced effect, achieved by day 11, was simulated with $k_{S}=0.00015 \mathrm{~min}^{-1}$

Fig. 3 are from a patient with cervical-dystonia-related complaints receiving BoNT/A intramuscularly (data from Fig. 1, injection series 1 in [18]). Starting at approximately day 11 , the maximum relief from symptoms was noted by the patient $\left(\mathrm{t}_{10}=10.6\right.$ days $)$. The rate constant $k_{S}$ was systematically varied from 0.0001 to $10 \mathrm{~min}^{-1}$ to provide an estimated value of $0.00015 \mathrm{~min}^{-1}$ for this model.

Other examples from two separate experimental studies using mice are also included in Fig. 3 [7,17]. Both sets of data following local intramuscular injections could be simulated $\left(k_{S}=0.001 \mathrm{~min}^{-1}\right)$. These data show a more rapid onset than the clinical data but are slower than the onset of paralysis seen with the in vitro rat NMJ data (see also Fig. 1). In this 4-step model, the NMJ data could be simulated with values of $k_{S} \geq$ $0.01 \mathrm{~min}^{-1}$.

A comparison was also made of the time courses for the different toxin species in the 3-step model for the NMJ and the 4-step model for the in vivo data examined here. As for the model tension prediction in Fig. 1, the numerical solution for the tension curve (Fig. 4, black line) was verified analytically (Fig. 4; white curve, Eqs. 2c and 3). As mentioned above, the 3-step NMJ model predicted that the time-to-10\% tension

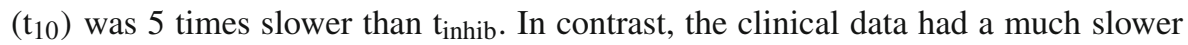
onset ( $\mathrm{t}_{10}=15,450 \mathrm{~min}$ or 10.6 days) for the BoNT-induced response, whose value

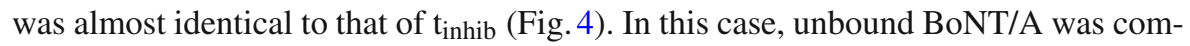
posed largely of the bulk species with a negligible presence of the free BoNT/A species. Thus, in the 4-step model for the clinical data, the time course of the unbound BoNT/A species was predicted to develop at essentially the same rate as the time course of paralysis. 


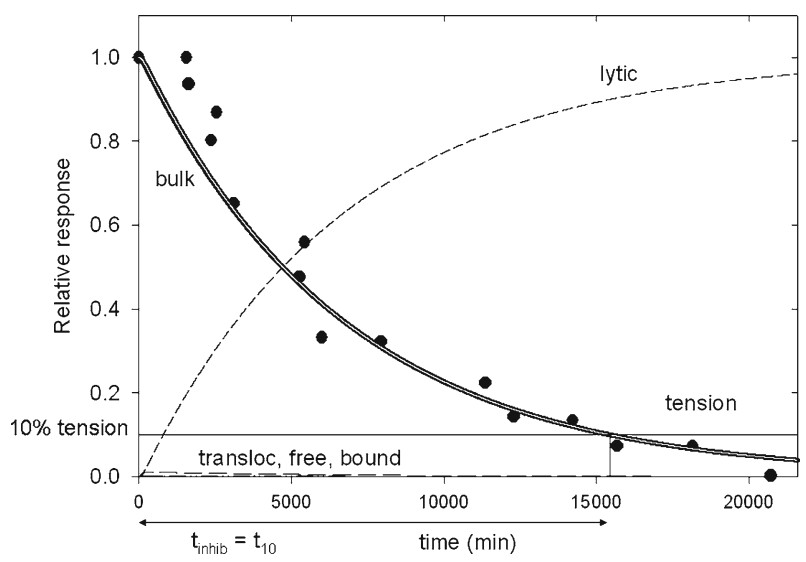

Fig. 4 Time-dependence of the BoNT/A species from the 4-step model that simulated the clinical data. The time courses of the different species can be compared with the relatively slow loss of tension $\left(\mathrm{t}_{10}=15,450 \mathrm{~min}\right.$ or 106 days) of BoNT/A-induced paralysis. In contrast to the in vitro NMJ (Fig. 1 ), the time courses of the unbound BoNT/A and the lytic species overlapped, making the estimated upper limit value of $\mathrm{t}_{\text {inhib }}$ longer in vivo than at the isolated NMJ. Closed circles: subjective clinical data from [18]; black line: responses were calculated numerically from the 4-step model using $\mathrm{k}_{S}=0.00015 \mathrm{~min}^{-1}$; white line: responses were calculated from Eq. $2 \mathrm{c}$

The area under the curve (AUC) calculations were done to compare the temporal profiles of the unbound species of BoNT using in vitro NMJ and in vivo human data. The areas for unbound BoNT/A are about the same ( $<20 \%$ difference) for the isolated $\mathrm{NMJ}$ and for the in vivo injections in man. The NMJ was exposed to $300 \mathrm{ng}$ while the patient received an i.m. injection of approximately $1 \mathrm{ng}$ BoNT/A. The amount of unbound BoNT at the NMJ decreased by $95 \%$ in $50 \mathrm{~min}$. In man, the same decrease occurred in about $20,000 \mathrm{~min}$ (400 times longer). The AUC for the NMJ is $5174 \mathrm{~min}-\mathrm{ng}$ using the 4-step model with $\mathrm{k}_{S}>0.01 \mathrm{~min}^{-1}$, while the AUC for man is $6406 \mathrm{~min}$ ng using the 4-step model with $\mathrm{k}_{S}=0.00015 \mathrm{~min}^{-1}$. As the value of $\mathrm{k}_{S}$ decreased, the amount of the unbound BoNT at any time point increased. Given the presumed differences in toxin purities and the uncertainty in the amount the patient received, these area values are remarkably similar. The rate of disappearance of the unbound BoNT/A is 400 times faster at the NMJ but this preparation also received about 300 times more BoNT/A than the patient. Thus, the calculated areas are similar due to offsetting differences in these rates and the amounts of toxin used.

To demonstrate the relation between the upper-limit estimate for $t_{\text {inhib }}$ and timeto- $10 \%$ tension ( $\mathrm{t}_{10}$ ), the values of $k_{S}$ were varied (Fig. 5). The symbols in this figure are the same as in Fig. 3 for representing $k_{S}$ values from the NMJ, from the i.m. injections in mice and from the clinical data. The 4-step model predicted that as $k_{S}$ decreased, the absolute values for $\mathrm{t}_{10}$ and $\mathrm{t}_{\text {inhib }}$ increased. The relations between $k_{S}$ and the upper-limit estimates of $t_{\text {inhib }}$ and times-to-10\% tension were non-linear (Fig. 5A). The largest component of the unbound BoNT/A species was predicted to be the bulk species for the data from the mouse local injections and from the clinical data where $k_{S} \leq 0.001 \mathrm{~min}^{-1}$. These calibration curves also provide upper-limit estimates for $\mathrm{t}_{\text {inhib }}$ that can be directly related to the duration for which a pre-defined threshold of 

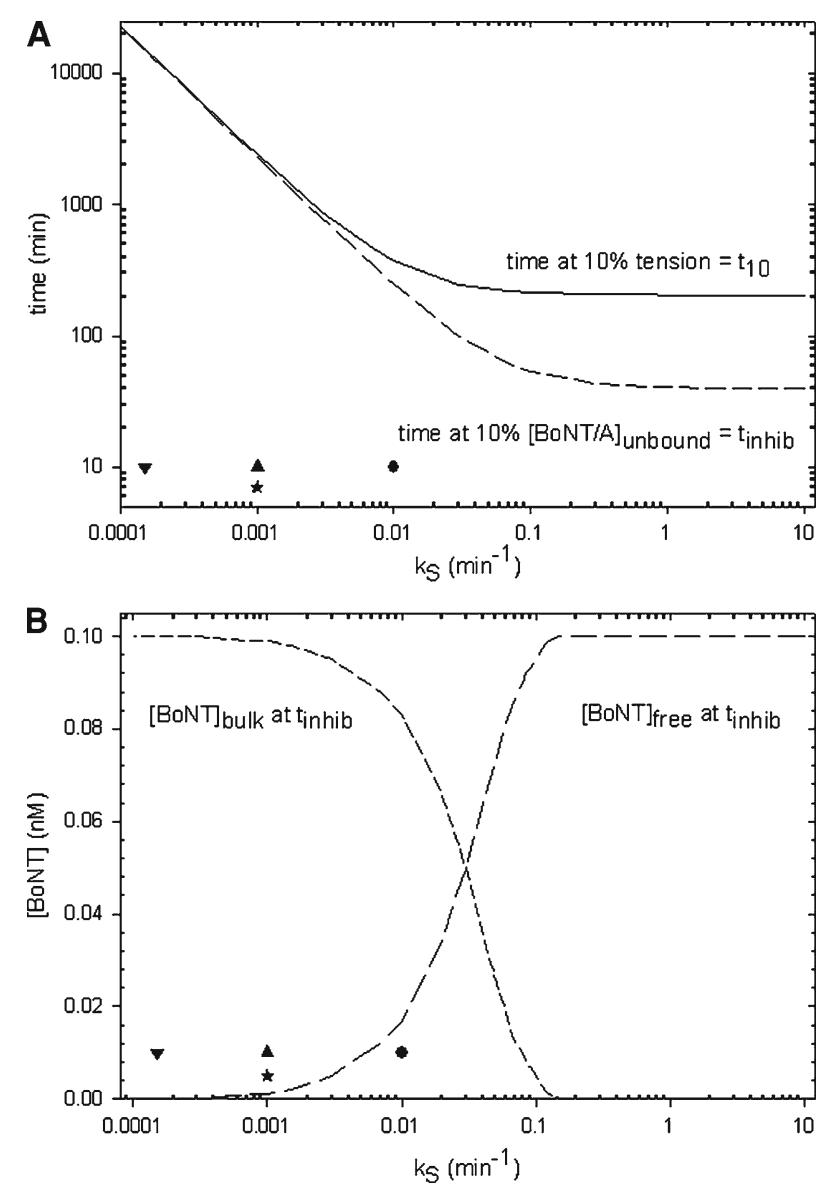

Fig. 5 Effects of varying $k_{S}$ on the upper-limit estimates of $t_{\text {inhib }}$ and onset times for BoNT/A-induced paralysis. Panel A: the two durations, the times-to- $10 \%$ peak tension $\left(\mathrm{t}_{10}\right)$ and the $\mathrm{t}_{\text {inhib }}$, increase and converge as the values of $k_{S}$ decrease. Panel B: the predicted concentration of unbound BoNT/A (decomposed into bulk and free species) at $\mathrm{t}_{\text {inhib }}$, is dependent on the value of $k_{S}$. The placement of symbols along the $x$-axis in both panels (upward triangles, downward triangles, stars, and circles) are defined in Fig. 3 and depict which of the unbound species and values of $k_{S}$ are associated with those simulations. For clarity, these symbols were placed just above the $x$-axis to correspond with the appropriate values of $\mathrm{k}_{S}$

tension is sustained. The plots in Fig. 5B over a range of values of $k_{S}$ show the relative, decomposed values for unbound BoNT/A at tinhib for the bulk and free species. The same trends were obtained with this model at other levels of post-exposure tension (50 and 90\%) that were examined (data not shown).

\section{Discussion}

The results of this study support the hypothesis that a minimal approach can model responses from in vitro and in vivo systems. This common tactic of using a reductionist approach was applied because computing the BoNT/A-induced perturbations in 
nerve-evoked muscle twitches is beyond the practicality of developing fully detailed models of the mechanisms of neurotoxin action upon vesicle-mediated chemical neurotransmission. Several implicit assumptions were made in the two models examined. Most importantly, it was assumed for computational convenience that the models were independent of compartments and that uniform distributions existed for all the BoNT/A species and binding sites. Despite these simplifications and our incomplete knowledge of cellular structures, relevant results emerged using biochemical observables as guides, specifically, the binding reaction rate [15] together with the microscopic binding and dissociation rate constants [19]. These values were consistent with classic methods in ligand-binding studies in which the inequality $[R]_{\text {tot }}>[T]_{\text {tot }}$ is assumed to exist within a well-stirred system that is far from a state of binding-site saturation [28]. Our calculations also show that various types of binding data from different tissues (central and peripheral neurons) and temperatures (4 to $\left.35-36^{\circ}\right)$ are related.

More insights were gained using the minimal models about the temporal relations between the extent of paralysis and the time that is available for an inhibitor to exert some effect, $t_{i n h i b}$, under different in vitro and in vivo conditions. These simulations predicted upper-limit estimates for $t_{\text {inhib }}$ during which $10 \%$ of the maximum amount of the unbound BoNT/A species is available for interacting with impermeable antagonists. Perhaps the most important concept to emerge from these minimal models is that as the onset rate of paralysis becomes slower, i.e., as the value of $k_{S}$ decreases, it also becomes a better indicator of the upper-limit for when $t_{\text {inhib }}$ and the times-to- $10 \%$ tension $\left(\mathrm{t}_{10}\right)$ converge (Fig. 5). The value of $\mathrm{t}_{\text {inhib }}$ is not a static amount of time but, rather, depends on the rate of paralysis onset.

These modeling results are related to a recent study of iatrogenic botulism. The existence of detectable serum levels of BoNT/A (12-24 mouse LD 50 ) in three out of four case-patients 2-3 days following extremely high i.m. doses (up to eight million mouse $\mathrm{LD}_{50}$ ) of research-grade toxin [29] suggests that the anti-toxin administration may have had a beneficial effect. These results also suggest that further development of animal models need to be conducted in vivo to test hypotheses that use onset rates of paralysis to obtain accurate estimates of $t_{\text {inhib }}$ for neutralizing antibodies or impermeable, low molecular weight inhibitors.

The binding step for BoNT/A was intentionally simplified because of the inherent complexity of the intoxication process and of the variety of reactions comprising neurotransmission. At least two molecular components form the functional binding site for BoNT/A, a protein and a ganglioside [14]. The ganglioside is GT1b or a related derivative $[30,31]$. Although the protein component (SV2, synaptic vesicle protein-2) has twelve putative transmembrane spanning regions [32,33], the location of the BoNT/A binding site is on a region of SV2 that is within a synaptic vesicle. This location implies that the interior solution of this structure must be in transient contact with the extracellular medium so that BoNT/A can bind immediately or bind following vesicle-pore closure and vesicle fission from the presynaptic membrane. During quiescent periods, the majority of the BoNT/A SV2 binding sites are not in contact with the extracellular medium and are unavailable for binding.

In contrast, with neural stimulation, these sites become exposed and available for binding, and as the frequency of stimulation increases, the time-to-10\% tension $\left(t_{10}\right)$ decreases [15] (Fig. 2). Although alterations in the other two reaction rates, by 
themselves, were insufficient to simulate the examined data, changes in all three of these reaction rates may be required to accurately model the experimental data. Thus, the enhanced rate of paralysis onset with increased nerve stimulation frequency may be a complicated function of enhanced calcium entry and an enhanced lytic effect, i.e., the proteolysis of the BoNT/A substrate SNAP25.

Other simplifications of the binding process in the 4-step model involve the initial step associated with the reaction rate, $k_{S}$. As noted in [15], there are anatomical and perhaps other barriers that interfere with BoNT/A readily diffusing to BoNT/A binding sites in the isolated NMJ. For the simulations of both the short-term in vitro and longer-term in vivo systems, delays in diffusion to binding sites caused by nonspecific binding [26,34] also need to be considered. Potential non-specific binders of BoNT/A include serum proteins (e.g., albumin) [4] and extracellularly located sites in the circulatory system.

Regarding the final two steps in the minimal models, simplifications were made for the translocation and lytic (toxic) processes $[13,15,35,36]$. The translocation step can be decomposed into at least a dozen steps, the most prominent being vesicle fission from the plasma membrane into the neuroplasm, the activation of energy-dependent proton pumps with the subsequent decrease in intra-vesicular $\mathrm{pH}$, the resultant conformational changes to BoNT/A, the reduction of its inter-chain disulfide bridge, the ion channel formation by the toxin's heavy chain, and the escape of the toxic moiety (presumably the $50 \mathrm{kD}$ light chain) into the neuroplasm where it exerts its toxic effect. A simplified single-step reaction is justified because the rates associated with many of these reactions are, as yet, undetermined.

The simplified lytic process includes among its most important reactions the zincdependent enzymatic activity of BoNT/A [15] that can be described by MichaelisMenten kinetics to cleave SNAP-25 [37]. This proteolysis prevents the formation of a functional SNARE protein complex (including synaptobrevin, syntaxin and other proteins) to block the release of acetylcholine and eventually block stimulus-evoked muscle contractions. The lytic step also contains a steep non-linear relation between the amount of substrate cleaved and the decrease of the evoked muscle twitch amplitude [38-40].

Although we selected a clinical study that had an abundance of relevant quantitative data [18], other clinical cases involving onset time scales of days should also be computationally examined and compared. For example, regarding cases of food-borne [41], iatrogenic and wound botulism [42] there is a lack of extensive clinical data sets in the publicly available literature and other open sources to archive paralysis time courses for future studies evaluating the temporal dependence of treatment effectiveness.

Notwithstanding the limitations of our minimal models, they have allowed us to simulate the onset kinetics of BoNT/A-induced effects observed in experimental and clinical studies. Beyond gaining a better appreciation of the temporal events that underlie the harmful as well as potentially beneficial effects of this family of neurotoxins, these models also provide us with an initial, quantitative framework for making extrapolations from animal model to human data.

Acknowledgements This paper is dedicated to our friend and colleague Dr Robert Sheridan. We thank Professor Lance L. Simpson for his insightful comments for an early version of this manuscript and to the 
referees for their valuable suggestions. The opinions or assertions contained herein are the private views of the authors and are not to be construed as official or as reflecting the views of the US Army or the US Department of Defense. This paper has been approved for public release with unlimited distribution. This work was supported by the Defense Threat Reduction Agency (DTRA JSTO-CBD project 3.10043_07_ RD_B to FJL) and the Computational Biology program of the DoD Advanced Research Project Agency (DARPA 05-0-DA-008 to FJL). KE held a National Research Council Research Associateship Award at the US Military Academy and the Army Research Laboratory. YC was sponsored by the US DoD High Performance Computing Modernization Program (HPCMP), under the High Performance Computing Software Applications Institutes (HSAI) initiative.

\section{References}

1. Arnon SS, Schechter R, Inglesby TV, Henderson DA, Bartlett JG, Ascher MS, Eitzen E, Fine AD, Hauer J, Layton M, Lillibridge S, Osterholm MT, O’Toole T, Parker G, Perl TM, Russell PK, Swerdlow DL, Tonat K (2001) Botulinum toxin as a biological weapon: medical and public health management. JAMA 285:1059-1070

2. Johnson EA, Bradshaw M (2001) Clostridium botulinum and its neurotoxins: a metabolic and cellular perspective. Toxicon 39:1703-1722

3. Simpson LL (2004) Identification of the major steps in botulinum toxin action. Annu Rev Pharmacol Toxicol 44:167-193

4. Ravichandran E, Gong Y, Al Saleem FH, Ancharski DM, Joshi SG, Simpson LL (2006) An initial assessment of the systemic pharmacokinetics of botulinum toxin. J Pharmacol Exp Ther 318:13431351

5. Adler M, Oyler G, Keller JE, Lebeda FJ (2000) Pharmacological countermeasures for botulinum intoxication. In: Somani SM, Romano JA (eds) Chemical warfare agents: toxicity at low levels. CRC Press, Boca Raton, pp 373-391

6. Millard CB (2005) Medical defense against protein toxin weapons. In: Lindler L, Lebeda FJ, Korch $\mathrm{G}$ (eds) Biological weapons defense: principles and mechanisms of infectious diseases and counterbioterrorism. Humana Press Inc, Totowa pp 255-283

7. Aoki KR (2004) Botulinum toxin: a successful therapeutic protein. Curr Med Chem 11:3085-3092

8. Public Law Project BioShield Act of 2004, 108-276, 118 Stat 835. Government Printing Office, Washington, DC (2004)

9. US Department of Health and Human Services, Project BioShield: Medical countermeasures for botulism (2007) http://www.hhs.gov/aspr/barda/mcm/botulism.html. Accessed 14 March 2008

10. US Department of Health and Human Services, HHS awards BioShield contract for botulism antitoxin (2006) http://www.hhs.gov/news/press/2006pres/20060601.html. Accessed 14 March 2008

11. Marks JD (2004) Deciphering antibody properties that lead to potent botulinum neurotoxin neutralization. Mov Disord 19(8):S101-108

12. Verderio C, Rossetto O, Grumelli C, Frassoni C, Montecucco C, Matteoli M (2006) Entering neurons: botulinum toxins and synaptic vesicle recycling. EMBO Reports 7:995-999

13. Lebeda FJ, Singh BR (1999) Membrane channel activity and translocation of tetanus and botulinum neurotoxins. J Toxicol Toxin Rev 18:45-76

14. Schiavo G, Matteoli M, Montecucco C (2000) Neurotoxins affecting neuroexocytosis. Physiol Rev $80: 717-766$

15. Simpson LL (1980) Kinetic studies on the interaction between botulinum toxin type A and the cholinergic neuromuscular junction. J Pharmacol Exp Ther 212:16-21

16. Aoki KR (2002) Botulinum neurotoxin serotypes A and B preparations have different safety margins in preclinical models of muscle weakening efficacy and systemic safety. Toxicon 40:923-928

17. Keller JE (2006) Recovery from botulinum neurotoxin poisoning in vivo. Neuroscience 139:629-637

18. Dressler D, Adib Saberi F (2005) Botulinum toxin: mechanisms of action. Eur Neurol 53:3-9

19. Dolly JO, Williams RS, Black JD, Tse CK, Hambleton P, Melling J (1982) Localization of sites for ${ }^{125}$ I-labelled botulinum neurotoxin at murine neuromuscular junction and its binding to rat brain synaptosomes. Toxicon 20:141-148

20. Segel IH (1975) Enzyme kinetics. Wiley-Interscience, New York

21. Adler M, Albuquerque EX, Lebeda FJ (1978) Kinetic analysis of end plate currents altered by atropine and scopolamine. Mol Pharmacol 14:514-529 
22. Hablitz JJ, Lebeda FJ (1985) Role of uptake in gamma-aminobutyric acid (GABA)-mediated responses in guinea pig hippocampal neurons. Cell Mol Neurobiol 5:353-371

23. Simpson LL (1974) Studies on the binding of botulinum toxin type A to the rat phrenic nervehemidiaphragm preparation. Neuropharmacology 13:683-691

24. Dressler D (2000) Botulinum toxin therapy. Georg Thieme Verlag, Stutgard

25. Sauro HM, Hucka M, Finney A, Wellock C, Bolouri H, Doyle J, Kitano H. (2003) Next generation simulation tools: the systems biology workbench and BioSPICE integration. OMICS 7:355-372

26. Daniels-Holgate PU, Dolly JO (1996) Productive and non-productive binding of botulinum neurotoxin A to motor nerve endings are distinguished by its heavy chain. J Neurosci Res 44:263-271

27. Hiromi K (1979) Kinetics of fast enzyme reactions. John Wiley and Sons, New York

28. De Lean A, Rodbard D (1979) Kinetics of cooperative binding. In: O'Brien RD (ed) The receptors: a comprehensive treatise. Plenum Press, New York, pp 143-192

29. Chertow DS, Tan ET, Maslanka SE, Schulte J, Bresnitz EA, Weisman RS, Bernstein J, Marcus SM, Kumar S, Malecki J, Sobel J, Braden CR (2006) Botulism in 4 adults following cosmetic injections with an unlicensed, highly concentrated botulinum preparation. JAMA 296:2476-2479

30. Yowler BC, Schengrund CL (2004) Botulinum neurotoxin A changes conformation upon binding to ganglioside GT1b. Biochemistry 43:9725-9731

31. Rummel A, Mahrhold S, Bigalke H, Binz T (2004) The HCC-domain of botulinum neurotoxins A and B exhibits a singular ganglioside binding site displaying serotype specific carbohydrate interaction. Mol Microbiol 51:631-643

32. Dong M, Yeh F, Tepp WH, Dean C, Johnson EA, Janz R, Chapman ER (2006) SV2 is the protein receptor for botulinum neurotoxin A. Science 312:592-596

33. Mahrhold S, Rummel A, Bigalke H, Davletov B, Binz T (2006) The synaptic vesicle protein 2C mediates the uptake of botulinum neurotoxin A into phrenic nerves. FEBS Lett 580:2011-2014

34. Wyman J, Gill SJ (1990) Binding and linkage: functional chemistry of biological macromolecules. University Science Books, Mill Valley

35. Adler M, Scovill J, Parker G, Lebeda FJ, Piotrowski J, Deshpande SS (1995) Antagonism of botulinum toxin-induced muscle weakness by 3,4-diaminopyridine in rat phrenic nerve-hemidiaphragm preparations. Toxicon 33:527-537

36. Koriazova LK, Montal M (2003) Translocation of botulinum neurotoxin light chain protease through the heavy chain channel. Nat Struct Biol 10:13-18

37. Schmidt JJ, Bostian KA (1997) Endoproteinase activity of type A botulinum neurotoxin: substrate requirements and activation by serum albumin. J Protein Chem 16:19-26

38. Jurasinski CV, Lieth E, Dang Do AN, Schengrund CL (2001) Correlation of cleavage of SNAP-25 with muscle function in a rat model of Botulinum neurotoxin type A induced paralysis. Toxicon 39:1309-1315

39. Kalandakanond S, Coffield JA (2001) Cleavage of SNAP-25 by botulinum toxin type A requires receptor-mediated endocytosis, pH-dependent translocation, and zinc. J Pharmacol Exp Ther 296: 980-986

40. Meunier FA, Lisk G, Sesardic D, Dolly JO (2003) Dynamics of motor nerve terminal remodeling unveiled using SNARE-cleaving botulinum toxins: the extent and duration are dictated by the sites of SNAP-25 truncation. Mol Cell Neurosci 22:454-466

41. Kongsaengdao S, Samintarapanya K, Rusmeechan S, Wongsa A, Pothirat C, Permpikul C, Pongpakdee S, Puavilai W, Kateruttanakul P, Phengtham U, Panjapornpon K, Janma J, Piyavechviratana K, Sithinamsuwan P, Deesomchok A, Tongyoo S, Vilaichone W, Boonyapisit K, Mayotarn S, Piya-Isragul B, Rattanaphon A, Intalapaporn P, Dusitanond P, Harnsomburana P, Laowittawas W, Chairangsaris P, Suwantamee J, Wongmek W, Ratanarat R, Poompichate A, Panyadilok H, Sutcharitchan N, Chuesuwan A, Oranrigsupau P, Sutthapas C, Tanprawate S, Lorsuwansiri J, Phattana N (2006) An outbreak of botulism in Thailand: clinical manifestations and management of severe respiratory failure. Clin Infect Dis 43:1247-1256

42. Jones JA, Salmon JE, Djuretic T, Nichols G, George RC, Gill ON, Brazier JS, Brett MM, Duerden BI, Fry NK, Hall V, Hope V, Lieftucht A, McLauchlin J, Pitcher DG, Weild A (2002) An outbreak of serious illness and death among injecting drug users in England during 2000. J Med Microbiol 51:978-984 